\title{
Identification of a time-varying mechanical system using the Akaike information criterion
}

\author{
M. RuzeK ${ }^{\mathrm{a}}$, S. Chesne And D. Remond \\ Université de Lyon, LaMCoS, CNRS UMR5259, INSA-Lyon, 69621 Villeurbanne, France
}

Received 8 December 2015, Accepted 8 November 2016

\begin{abstract}
This article deals with the use of Akaike information criterion in the case of an identification problem of a time varying mechanical system. We studied a prismatic beam with a disk excited in torsion to illustrate how the proposed approach can be implemented. The boundary conditions of the beam can change in a controlled way during the experiment. Therefore, our system can be considered as a one d.o.f. system with time-varying parameters. A method based on least-square estimates is used for the identification of the parameters. The use of the Akaike information criterion allows to choose automatically the order of polynomial basis used during the estimation step of the derivatives of the measured angle signal. It is shown through an experimental validation that the AIC criterion is a robust and automatic tool for numerical estimation of signal derivatives.
\end{abstract}

Key words: Information criteria / inverse problems / identification

\section{Introduction}

In many engineering problems we are interested in identifying some real physical system. In other words, we want to identify a mathematical model which would describe the physical phenomenon, which we observe. In this article, we deal with the identification of a simple mechanical system with one degree of freedom and one time-varying parameter.

The systems with time-varying parameters can be found in many physical problems. For example, the mechanics of biological and human tissues and joints can be described by time-varying models [1]. In the same works, the robotics and joystick command systems are also considered as time-varying mechanical systems. The identification of the time-varying systems has been studied by different approaches: by the ensemble average method [2], by a parallel-cascade algorithm [3], or by a wavelet method [4]. Numerous techniques use the projection of the time-varying parameters on an orthogonal basis of functions $[5,6]$. The choice of this basis can be a bit arbitrary, there were methods using Legendre polynomials $[7,8]$, block-pulse functions [9], Fourier series [10], Laguerre polynomials [11], Chebyshev polynomials [12$14,14-17]$ or classical polynomial basis $[18,19]$. In principle, all of these methods work quite well. However, their

\footnotetext{
${ }^{a}$ Corresponding author: michal.ruzek@insa-lyon.fr
}

weakness is the choice of the size of the functional basis. Indeed, we have no clue how large the basis of polynomial functions should be. Most often, one must use some iterative scheme and see how the results look like. Then, some human judgement is necessary to choose a good size of the basis of polynomial functions. This human factor makes these methods a bit cumbersome and time-costly.

In this article, we propose to apply a simple statistical tool called Akaike information criterion (AIC) to choose a suitable polynomial basis automatically. We deal with the same experimental data as described in [17]. The AIC criterion was developed by Akaike [20] in 1974. Its goal is to choose the optimal model from a set of candidate models and some observation. It was widely used in statistics and applied in many real-life problems, mostly in biology [21-24]. So far, its use for the mechanical problems was very limited. Its use for a choice of vibration models was proposed in [25]. It was also used together with autoregressive moving average method to identify the location of a damage element in civil engineering in [26].

This article is organized as follows. Section 2 describes the experimental set-up and measured data. Section 3 describes the technique of identification of the model and presents the results. A comparison with the Bayesian information Criterion (BIC) is briefly presented. Section 4 concludes the article and Appendix describes the information criteria used in this study. 


\section{Experimental set-up}

To build an accurate and easy to used mechanical system with time varying parameters is not so easy. The experimental set-up can be seen in Figure 1. It consists of a triangular prism mounted vertically. At the lower end of the bar, there is a disk which serves as an inertia. At the upper end of the prism, there is a slider. This slider serves as a clamped boundary condition, but it can move freely up and down during the experiment. This motion changes the boundary condition and, consequently, changes also the torsional stiffness of the prismatic beam. If we know the position of the slider in time, we are able to calculate the theoretical torsional stiffness of the beam in time. This is very useful, because we can compare this time-varying stiffness to the stiffness identified by our method.

Finally, the excitation is achieved via an electric motor which is connected to the disk through the torque cell and rotation accelerometer. During the experiment, we are able to measure simultaneously the driving torque, the rotation angle and the position of the slider. A typical measure is shown in Figure 2. We note that the torque signal is by far the noisiest one. This is probably due to some micro-gaps in the screwing mechanism and maybe due to the influence of the flexural vibration of the beam. On the other hand, the rotation angle signal is very neat, which is necessary because we will be dealing with estimation derivatives of this signal. The sampling frequency of the measure was $1000 \mathrm{~Hz}$. More information about this set up can be found in [17].

\section{Identification of the system}

The system described in Section 2 can be approximately described as a system with one (rotational) degree of freedom. Its equation of motion can be written as

$$
I \ddot{\theta}+C \dot{\theta}+K \theta=T
$$

where $I$ is the global inertia of the beam and the disk, $C$ is the damping coefficient, $K$ is the rotational stiffness, $\theta(t)$ is the rotation angle and $T(t)$ is the driving torque. In our case, we want to identify the parameters $I, C$ and $K$ while we measure $\theta(t)$ and $T(t)$. In previous studies [17-19], the torsional inertia and damping were considered constant in time, but the torsional stiffness is necessarily a function of time $K=K(t)$. In this paper, the three mechanical parameters are considered as time-varying.

The process of system identification can be separated into two parts as shown in Figure 3. Firstly, the first and second time derivatives of the angle $\theta$ are estimated. This is done using the polynomial fitting and the AIC criterion. Secondly, once all the derivatives are estimated, the optimal parameters $I, C$ and $K$ are identified in order to satisfy the equation of motion given by (1). Both of these procedures are detailed in the following.

\subsection{Estimation of derivatives}

The heart of the problem of identifying the parameters of the Equation (1) lies in the fact that we only measure the angle $\theta$. The time derivatives of this angle are unknown and we need to estimate them from $\theta$. There are many methods for the estimation of derivatives. Here, we opt for the polynomial regression optimized by the AIC criterion.

Let us look the derivative of $\theta$ at some time $t_{\mathrm{i}}$. We suppose that we dispose the measurement of $\theta$ for the neighboring times to $t_{\mathrm{i}}$. In a small interval of interest we dispose $2 \mathrm{~N}+1$ measures of $\theta$. We can put them in the vector $\theta=\left[\theta\left(t_{\mathrm{i}-\mathrm{N}}\right), \ldots, \theta\left(t_{\mathrm{i}}\right), \ldots, \theta\left(t_{\mathrm{i}+\mathrm{N}}\right)\right]^{\mathrm{T}}$.

In this small interval around the time $t_{\mathrm{i}}$ the function $\theta(t)$ can be developed in the Taylor series as

$$
\theta(t)=\sum_{j=0}^{n} \alpha_{j}\left(t-t_{i}\right)^{j}
$$

The coefficients $\alpha$ of the expansion given by (2) can be easily obtained by the least-square linear fit of the measured vector $\theta$ by the Taylor series decomposition. However, how should we choose the number of polynomials $n$ in the Taylor series decomposition? This is the main problem of many identification methods using an expansion process.

The AIC criterion can answer this question (definition of the AIC criterion can be found in the appendix). Let us consider a small interval of time and the corresponding measure of angle $\theta$ (see Fig. 4a). We can fit this function by Taylor series with different maximal polynomial order $n$. The optimal fit should be the one which minimizes the value of the AIC function. According to the Figure 4b, the Taylor series with $n=16$ is the optimal fit for the measured function. However, to highlight the main interest of this kind of criterion, if we add artificially some uncorrelated noise as it is shown in Figure $5 \mathrm{a}$, than the AIC criterion would choose the optimal model among the Taylor series the one having $n=7$ (Fig. 5b). Practically, that means that polynomial function of higher order would be used to fit the noise.

The strength of this approach is its robustness and automatism. A simple program can be constructed to automatically choose the model (Taylor series) having the lowest value of AIC among all the other models.

Once the optimal model (Taylor series) is identified via AIC criterion, it can be easily derived to obtain the first and second derivatives. In a certain point of view, the AIC avoids an unwanted expansion of the noise on the polynomial basis. These expansions usually disturb drastically the derivation step.

It should be noted that the maximal number of polynomials (so-called candidate models) has to be high enough to represent well the fitted model. The obvious question is, however, what is enough? Let us see an example represented in Figure 4. Here, we use the maximal polynomial degree 19 for all the measured intervals. If the minimum AIC value had occurred for polynomial with 

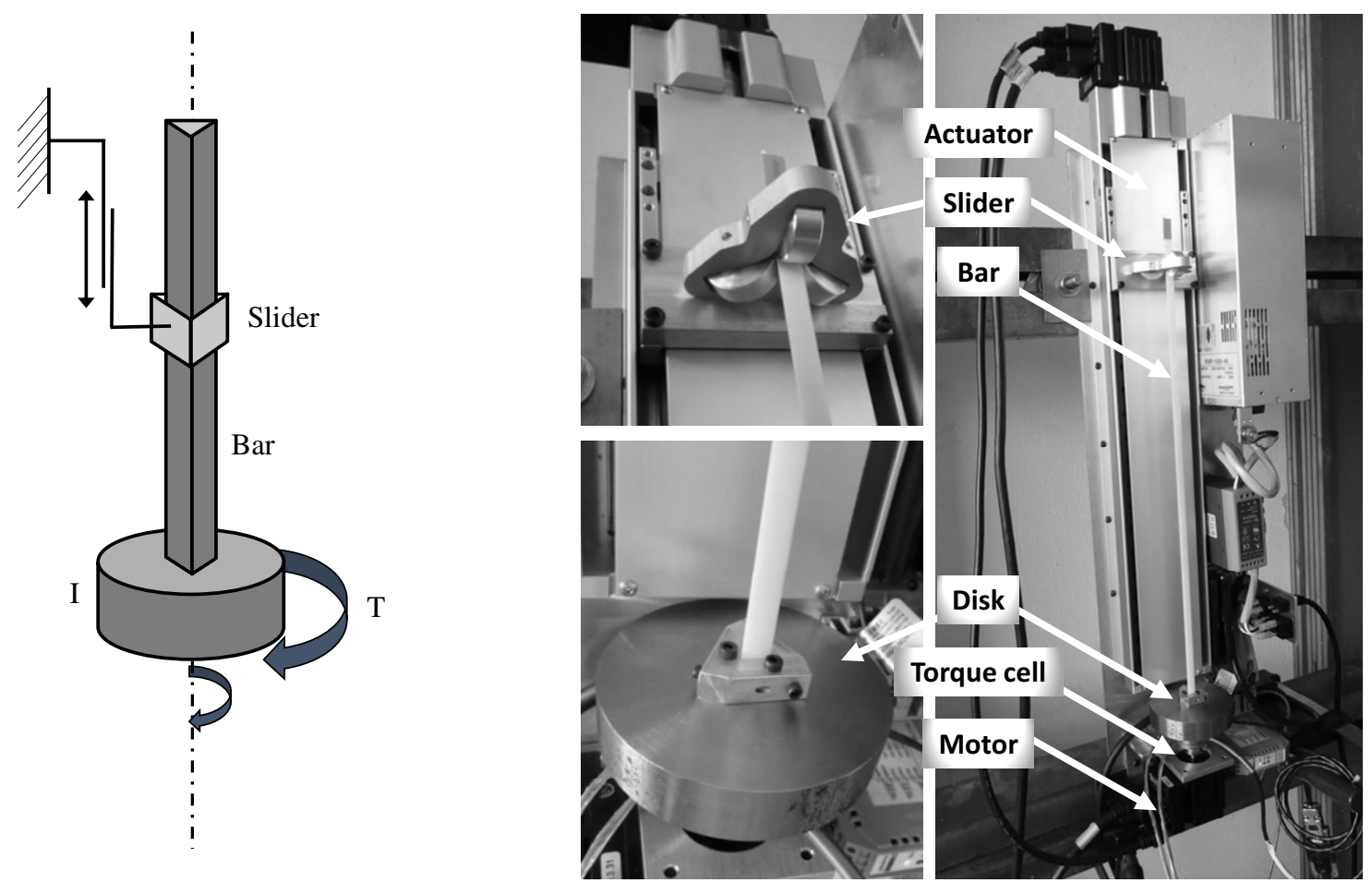

Fig. 1. Representative scheme and the experimental mounting of the prism beam in torsion.

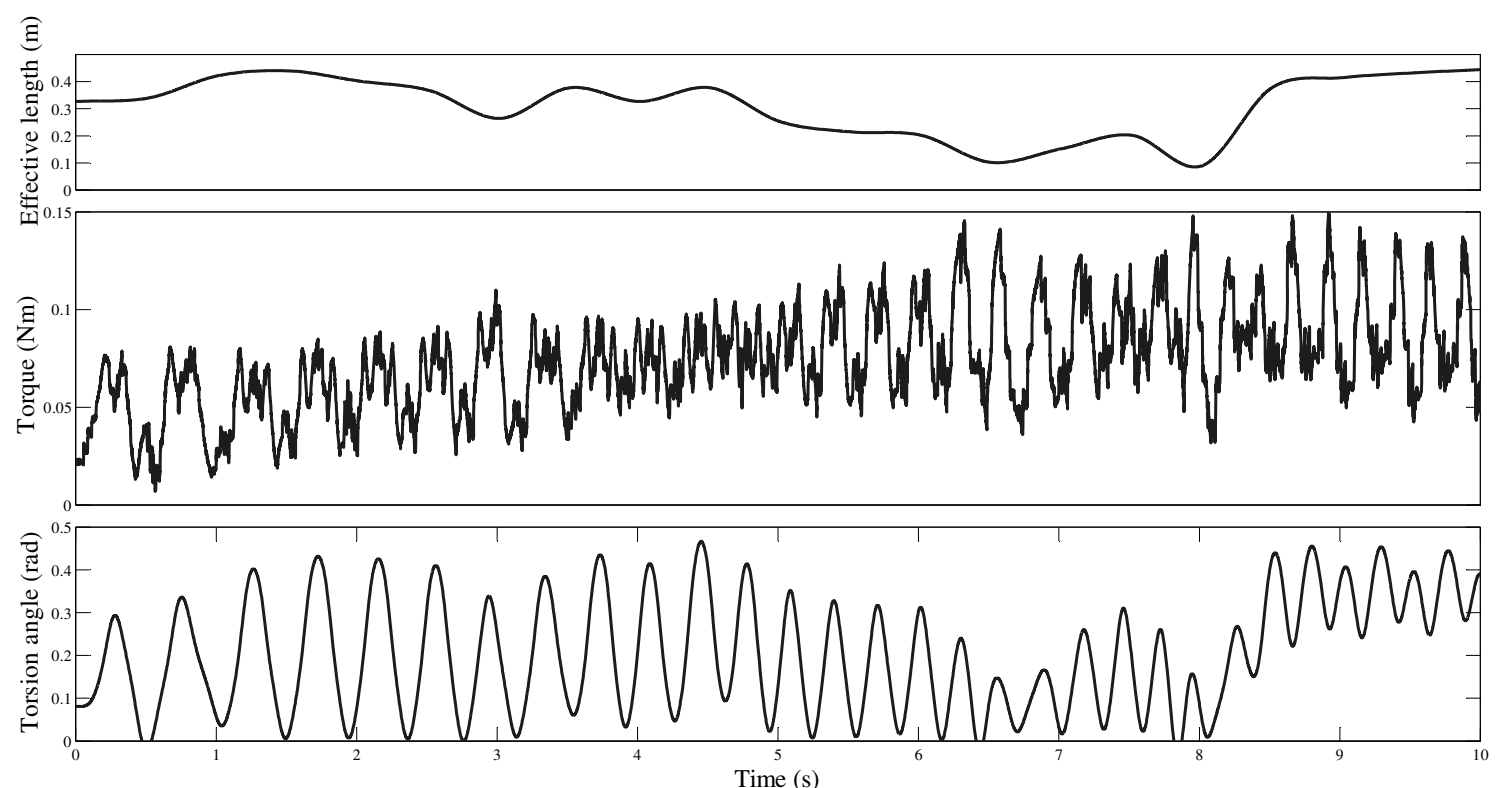

Fig. 2. A typical measure obtained from our experimental setup: above - the slider position, in the middle - the torque applied, below - rotation angle.

$n=19$, then the maximum polynomial degree 19 might not have been enough, because for $n=20$, we might have got lower value of AIC. However, if we got the minimal value of AIC for $n=16$ (see Fig. 4b), then there is very low risk, than adding $n=20$ to our candidate models would change anything. Therefore, it is recommended that the optimal model should not be the first neither the last model among the candidate models.
For comparative purpose, Bayesian Information Criterion is also plotted in Figures 4 and 5 . It can be seen that the results and conclusions are very similar.

\subsection{Identification of parameters}

Once the derivatives of $\theta$ are identified for all measured points, we can identify the optimal parameters of 
M. Ruzek et al.: Mechanics \& Industry 18, 203 (2017)

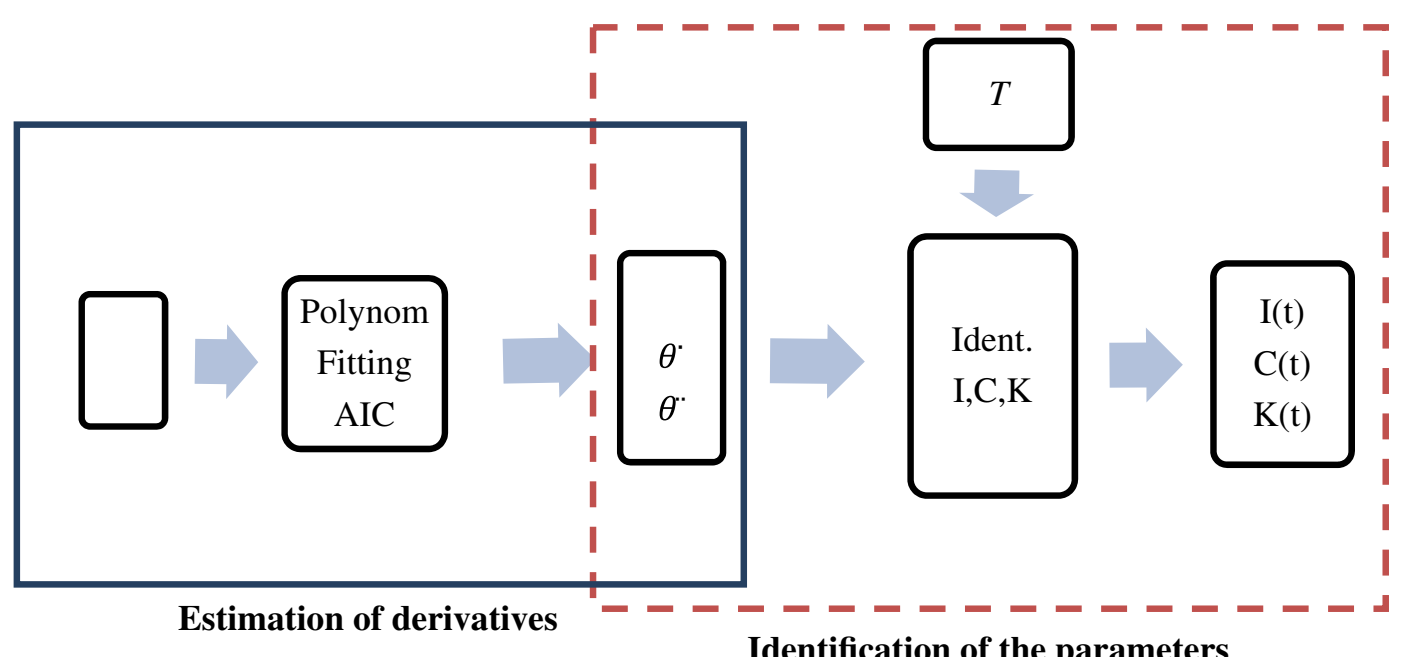

Fig. 3. The steps of the identification method.
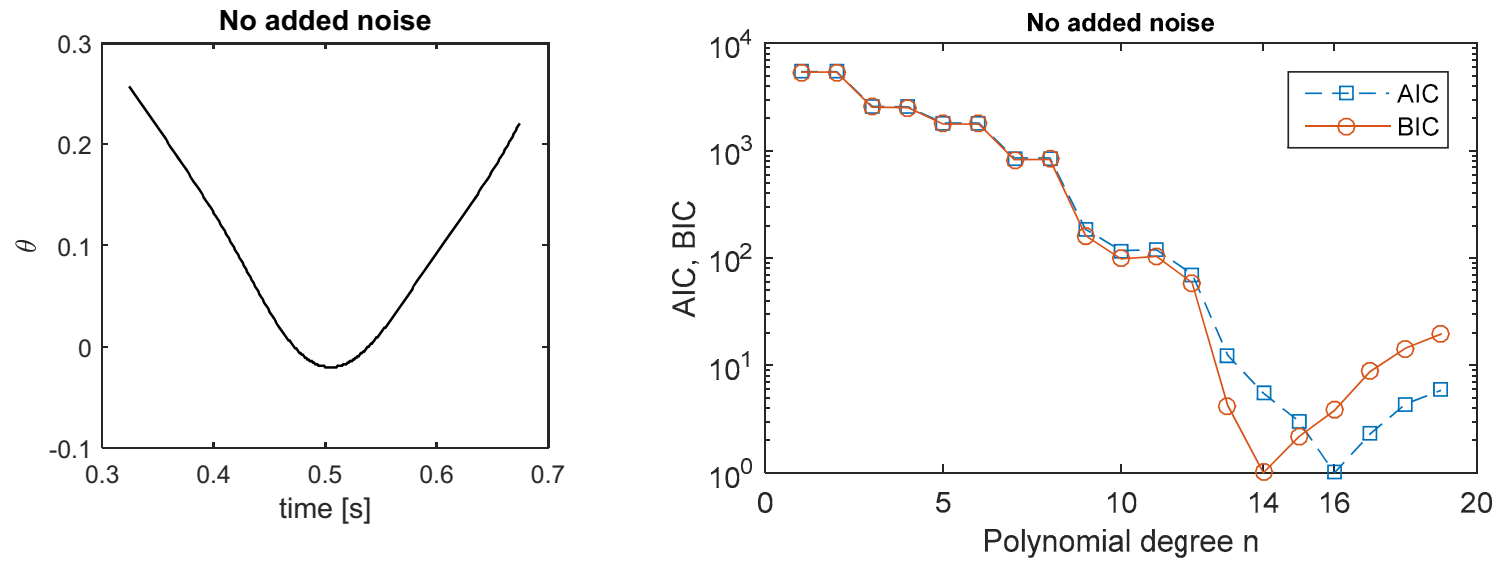

Fig. 4. Example of the use of AIC and BIC to fit measured data. (a) By fitting an experimental curve with low noise, (b) AIC and BIC favors high polynomial fit.
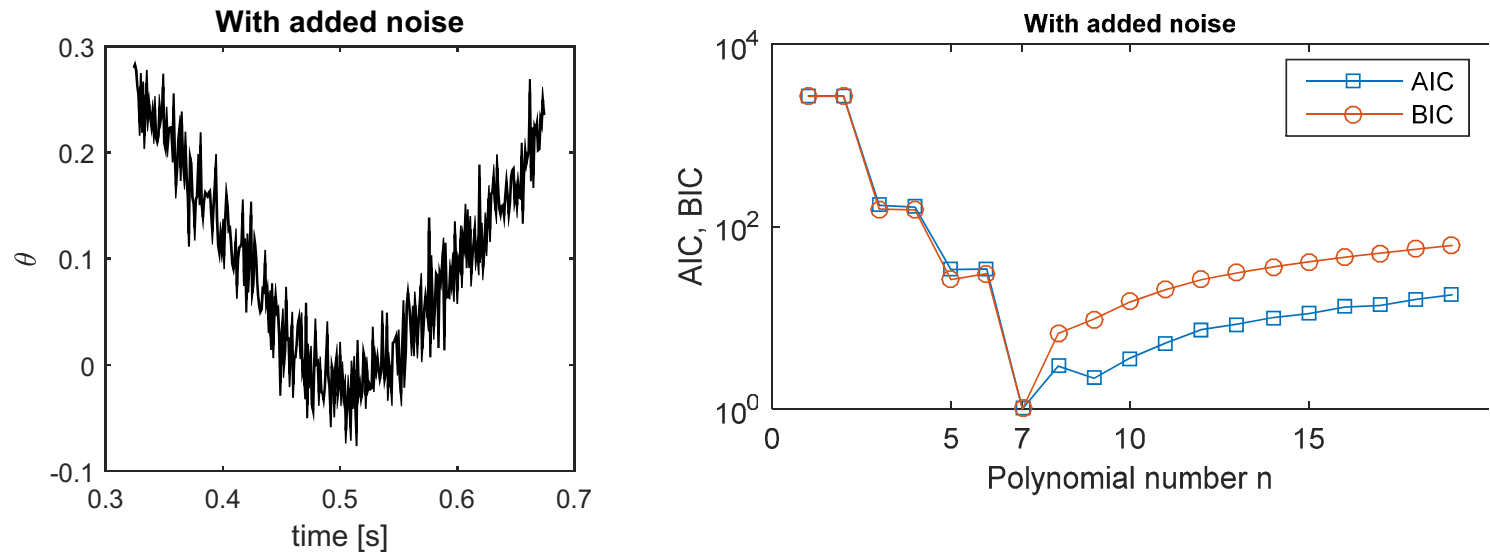

Fig. 5. Example of the use of AIC and BIC to fit a noisy curve. (a) By fitting the same curve with artificially added noise, (b) $\mathrm{AIC}$ and BIC favor lower degree polynomials. 
the equation of motion (1). For the $i$-th measured time $t_{i}$, we can write the Equation (1) as

$$
I \ddot{\theta}\left(t_{i}\right)+C \dot{\theta}\left(t_{i}\right)+K \theta\left(t_{i}\right)=T\left(t_{i}\right)
$$

This equation cannot be solved for $I, C$ and $K$, because we have more unknowns than equations. On the other hand, we can assume that the variation of these parameters is much slower than the sampling frequency of the time. Under these assumptions, we can consider the values of $I, C$ and $K$ constant over some short interval of time. According to the method used in Section 3.1, we consider that we dispose $2 \mathrm{~N}+1$ values of $\theta, \dot{\theta}, \ddot{\theta}$ in the vicinity of time $t_{i}$. Then, we can write a system of linear equations for the parameters $I, C$ and $K$ at the time $t_{i}$

$$
\left[\begin{array}{ccc}
\ddot{\theta}\left(t_{i-N}\right) \dot{\theta}\left(t_{i-N}\right) & \theta\left(t_{i-N}\right) \\
\vdots & \vdots & \vdots \\
\ddot{\theta}\left(t_{i}\right) & \dot{\theta}\left(t_{i}\right) & \theta\left(t_{i}\right) \\
\vdots & \vdots & \vdots \\
\ddot{\theta}\left(t_{i+N}\right) \dot{\theta}\left(t_{i+N}\right) & \theta\left(t_{i+N}\right)
\end{array}\right] *\left[\begin{array}{c}
I \\
C \\
K
\end{array}\right]=\left[\begin{array}{c}
T\left(t_{i-N}\right) \\
\vdots \\
T\left(t_{i}\right) \\
\vdots \\
T\left(t_{i+N}\right)
\end{array}\right]
$$

Where the parameters $I, C$ and $K$ correspond to the time point $t_{i}$. Parameters $I, C$ and $K$ can be easily obtained from Equation (4) by pseudo-inversing the matrix of the system. The number of equations to take into account into the system (4) is a bit arbitrary. More equations we take into account, smoother is the solution, but this filtering property can badly reconstruct fast variations of the identified parameters... It is possible to use some iterative technique to find a suitable number of equations to use. However, the influence of the number of equations in (4) is not so important in the studied case.

In Figure 6, there are identified parameters $I, C$ and $K$ obtained by the method described above. The number of equations in the system (4) was 200, which means physically that these parameters were considered constant over $0.2 \mathrm{~s}$ (the sampling frequency was $1000 \mathrm{~Hz}$ ). We note that the identified inertia parameter is noisy, which is probably due to its link with the second derivative, which is much more sensitive to noise than the others. On the other hand, the stiffness corresponds well to the theoretical stiffness parameter obtained from the measured position of slider (see Fig. 2 above). Inertia and stiffness was the two main parameter of this study. They are well reconstructed, specially the variation of the stiffness. The damping of the real system is very low. In previous studies [17-19] it has been set to zero without disturbing drastically the identification of the time varying stiffness. The identified damping coefficient here is very small, but it cannot be compared to other independent measure. Nevertheless the mean value of this coefficient over the time domain is positive, which is in agreement with the physics of the system. The identification is generally bad at the beginning and the end of the measure. This is due to erroneous derivative estimation at the borders.

\section{Conclusion}

In this article, an inverse method using on the Akaike information criterion is presented. This method enables us to estimate the temporal derivatives of a measured signal and consequently to identify the parameters of a mechanical system. It has been illustrated with a single degree of freedom system. The AIC criterion is used to choose automatically the optimal polynomial for fitting locally a measured signal which is of great interest for this kind of identification problem. The advantage of this approach is the robustness of this method. It can deal with different level of noise and automatically choose the optimal polynomial model, contrary to previous developed methods [17-19]. The BIC criterion was also briefly investigated and it gave us almost identical results.

Three parameters of the single degree of freedom system were determined: inertia, damping and stiffness coefficients. While the identified damping parameter cannot be verified independently, the identified inertia and stiffness coefficients correspond well to other independent measures (see [17]).

The proposed approach could be applied to other identification problems. One perspective is the identification of the systems with a few degrees of freedom. Another perspective would be to increase the accuracy of the identification by making constant (but still unknown) some of the non-varying parameters ( $\mathrm{I}$ and $\mathrm{C}$ in this case).

\section{Appendix: Akaike and Bayesian information criteria}

The Akaike information criterion was introduced by Akaike in [20]. A more recent critical review is proposed by Burnham and Anderson in [21]. In a simplified version the problem solved by the AIC criterion is as follows. Let us have a system of $N$ (generally nonlinear) equations

$$
f_{i}\left(M_{j}\left(p_{j}\right)\right)=d_{i}, \quad i=1 \ldots N
$$

where the coefficient $i$ represents different independent measures $d_{i}$. The $i$-th value of the model $M_{\mathrm{j}}$ is described by the function $f_{i}\left(M_{j}\left(p_{j}\right)\right)$. This function depends on some vector of parameters $p_{j}$, which is to be determined. This parameter vector can be determined by the nonlinear least squares, in other words by minimizing the sum of residuals $R_{j}=\sum_{i} \varepsilon_{i j}^{2}$, where

$$
\varepsilon_{i j}\left(p_{j}\right)=f_{i}\left(M_{j}\left(p_{j}\right)\right)-d_{i}
$$

The optimal least-square estimate of the parameter $\hat{p}_{j}$ for the model $j$ is obtained as

$$
\hat{p}_{j}=R_{j}\left(p_{j}\right)
$$

The AIC function for a model $j$ is defined as follows

$$
A I C_{j}=N \ln \sigma_{j}^{2}+2 K_{j}+\frac{2 K_{j}\left(K_{j}+1\right)}{N-K_{j}-1}
$$



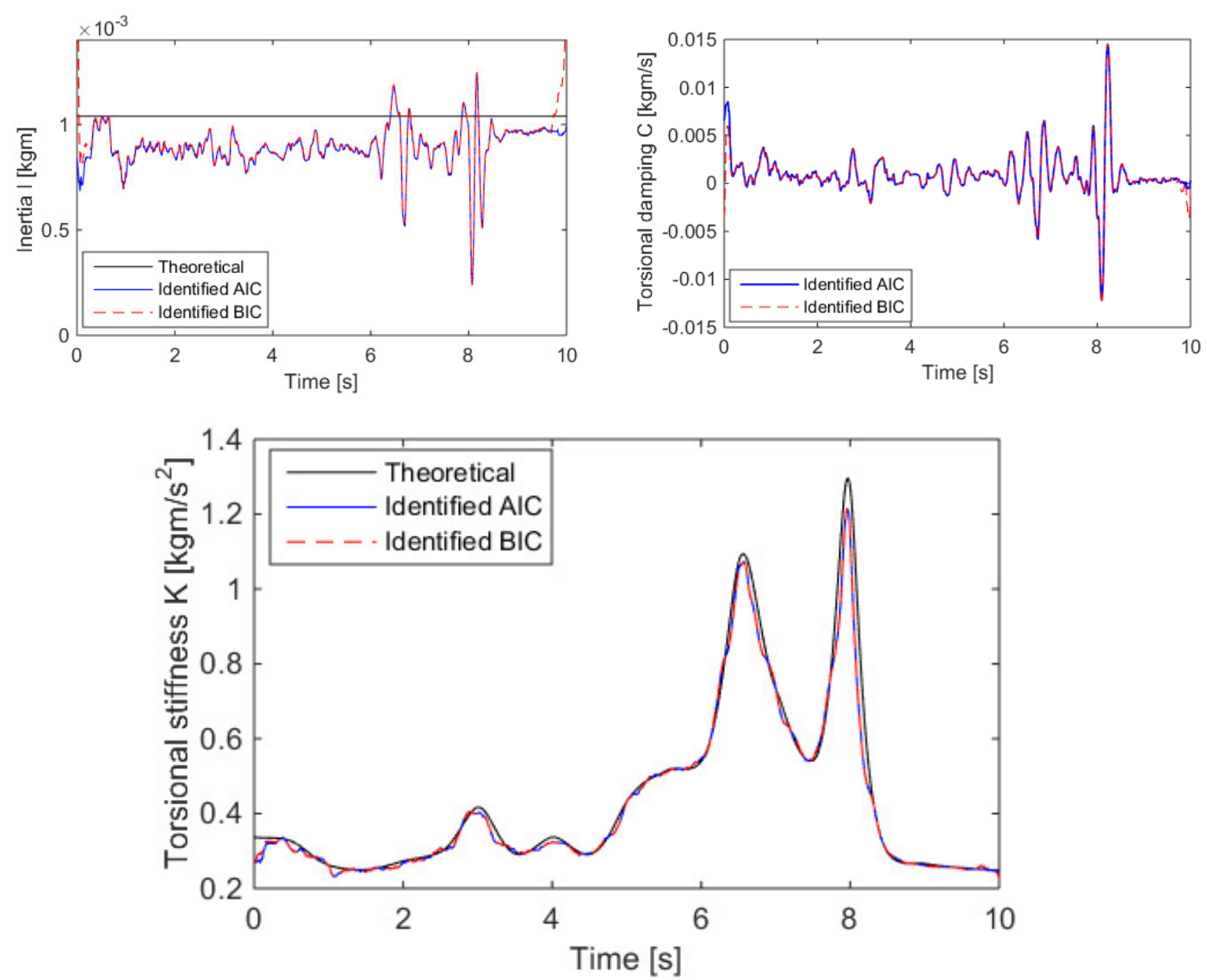

Fig. 6. Parameters of the time-varying system identified by the inverse method. Above on the left: the identified torsional inertia (blue when using AIC and red with BIC) compared to the theoretical inertia (black). Above on the right: the identified torsional damping coefficient. Below: the identified torsional stiffness compared to the stiffness obtained from the slider position.

where $K_{j}$ is the number of elements in the vector of parameters $p_{j}$, and $\sigma_{j}$ is the estimate of the standard deviance of the measure using the model $j$ :

$$
\sigma_{j}^{2}=\frac{R_{j}\left(\hat{p}_{j}\right)}{N-1}
$$

Under the assumptions that the measures $d_{i}$ are independent identically normally distributed random variables, the AIC function is an unbiased estimate of the KullbackLeibler distance between the measure and the model. In practice, we want this distance to be minimal; we want the model to be as close to the reality as possible. This means, that we search for the model with the lowest value of AIC. In order to compare different models, we must choose some candidate models at first. In our case, these candidate models are simple polynomials of the Taylor series, but these models can be represented by any function. Then, the optimal parameters $\hat{p}_{j}$ of the models are determined by the least-squares and the minimal residuals (A.5) are evaluated. The model chosen by the AIC criterion is not simply the model having the lowest residuals. It is a model which has a low residual but a low number of parameters as well.

AIC criterion selects a model which is optimal in a sense of the prediction. It means that the selected model would give the best prediction of unmeasured data.
Unlike the AIC the Bayesian information criterion (BIC) is does not identify the best predictive model from the candidate models, but the model which is the most probable. Quite surprisingly, under the same hypothesis which were used for the AIC, the BIC function looks rather similar to AIC (see details in [21]):

$$
B I C_{j}=N \ln \sigma_{j}^{2}+K_{j} \ln N
$$

In most cases the BIC and AIC model selections give similar results.

\section{References}

[1] R.E. Kearney, I.W. Hunter, System identification of human joint dynamics, Critical Reviews in Biomedical Engineering 18 (1990) 55-87

[2] M. Baker, Y. Zhao, D. Ludwig, R. Wagner, R.E. Kearney, Time-varying Parallel-cascade System Identification of Ankle Stiffness from Ensemble Data, Proceedings of the $26^{\text {th }}$ Annual international conference of the IEEE EMBS, San Francisco, California, United States, $1^{\text {st }}-5^{\text {th }}$ September 2004, pp. 4688-4691

[3] D. Ludwig, T.S. Visser, H. Giesbrecht, R.E. Kearney, Identification of Time-Varying Intrinsic and Reflex Joint Stiffness, IEEE Trans. Biomedical Eng. 58 (2011) 17151723 
[4] X. Xu, Z.Y. Shi, Q. You, Identification of linear time-varying systems using a wavelet-based state-space method, Mech. Systems Signal Process. 26 (2012) 91-103

[5] S.G. Mouroutsos, P.N. Paraskevopoulos, Identification of time-varying linear systems using orthogonal functions, J. Frankl. Inst. 320 (1985) 249-258

[6] T.S. Morais, V. Steffen, N. Bachschmid, Time-varying parameter identification using orthogonal functions, J. Phys. Conf. Ser. 135 (2008) 102072

[7] J.H. Chou, I.R. Horng, Identification of time-varying bilinear systems using Legendre series, J. Frankl. Inst. 322 (1986) 353-359

[8] C. Hwang, T.Y. Guo, Parameter identification of a class of tie-varying systems via orthogonal shifted Legendre polynomials, J. Frankl. Inst. 318 (1984) 56-69

[9] C. Hwang, T.Y. Guo, Identification of lumped linear time-varying systems via block-pulse functions, Int. J. Cont. 40 (1984) 571-583

[10] M. Razzaghi, S.D. Lin, Identification of time-varying linear and bilinear systems via Fourier series, Comp. Elec. Eng. 17 (1991) 237-244

[11] P.R. Clement, Laguerre functions in signal analysis and parameter identification, J. Frankl. Inst. 313 (1982) 8595

[12] S.V. Lapin, Identification of time-varying nonlinear systems using Chebyshev polynomials, J. Comp. Appl. Math. 49 (1993) 121-126

[13] Y.P.Shih, C.C.Liu, Parameter estimation of time-varying systems via Chebyshev polynomials of the second kind, Int. J. Sys. Sci. 17 (1986) 459-464

[14] P.N. Paraskevopoulos, Chebyshev series approach to system identification, analysis and optimal control, J. Frankl. Inst. 316 (1983) 135-157

[15] D. Rémond, J. Neyrand, G. Aridon, R. Dufour, On improved use of Chebyshev expansion for mechanical system identification, Mech. Sys. Signal Proc. 22 (2007) 390-407
[16] C. Chochol, S. Chesné, D. Rémond, An original differentiation tool for identification on continuous structures, J. Sound Vib. 332 (2013) 3338-3350

[17] F. Martel, D. Rancourt, C. Chochol, Y. St-Amant, S. Chesné, D. Rémond, Time-varying torsional stiffness identification on a vertical beam using Chebyshev polynomials, Mech. Sys. Signal Proc. 54-55 (2015) 481-490

[18] S. Chesné, F. Martel, C. Chochol, D. Rancourt, D. Rémond. Identification of time varying stiffness using derivative estimator and polynomial basis. ISMA International conference on Noise and Vibration Engineering, Sep 2014, LEUVEN, Belgium, 2014

[19] S. Chesné, C. Chochol, D. Rémond, F. Martel, D. Rancourt. Identification of time varying parameters using numerical differentiation by integration, 21st International Congress on Sound and Vibration, Jul 2014, Beijing, China. pp. 1-8, (2014).

[20] H. Akaike, A new look at the statistical model identification, IEEE Trans. Automatic Control 19 (1974) 716-723

[21] K.P. Burnham, D.R. Anderson, Model selection and multimodel inference, Springer, New York, 2002

[22] M.A. Butler, A.A. King, Phylogenetic comparative analysis: A modeling approach for adaptive evolution, Am. Naturalist 164 (2004) 683-695

[23] B.C. O'Meara, C. Ane, M.J. Sanderson, P.C. Wainwright, Testing for different rates of continuous trait evolution using likelihood, Evolution 60 (2006) 922-933

[24] D. Posada, K.A. Crandall, Selecting the best-fit model of nucleotide substitution, Systematic Biology 50 (2001) 580-601

[25] M. Ruzek, J.L. Guyader, C. Pézerat, Information criteria and selection of vibration models, J. Acoust. Soc. Am. 136 (2014) 3040-3050

[26] J.B. Bodeux, J.C. Golinval, Application of ARMAV models to the identification and damage detection of mechanical and civil engineering structures, Smart Mater. Struct. 10 (2001) $1-10$ 